\title{
Verified Interval Enclosure Techniques for Robust Gain Scheduling Controllers
}

\author{
Julia Kersten, ${ }^{a}$ Andreas Rauh, ${ }^{a}$ and Harald Aschemann ${ }^{a}$
}

\begin{abstract}
In real-life applications, dynamic systems are often subject to uncertainty due to model simplifications, measurement inaccuracy or approximation errors which can be mapped to specific parameters. Uncertainty in dynamic systems can come either in stochastic forms or as interval representations. The latter is applied if the uncertainty is bounded as it will be done in this paper. The main idea is to find a joint approach for an interval-based gain scheduling controller while simultaneously reducing overestimation by enclosing state intervals with the least amount of conservativity. The robust and/ or optimal control design is realized using linear matrix inequalities (LMIs) to find an efficient solution and aims at a guaranteed stabilization of the system dynamics over a predefined time horizon. A temporal reduction of the widths of intervals representing worst-case bounds of the system states at a specific point of time should occur due to asymptotic stability proven by the employed LMI-based design. However, for commonly used approaches in the computation of interval enclosures, those interval widths seemingly blow up due to the wrapping effect in many cases. To avoid this, we provide two interval enclosure techniques - an exploitation of cooperativity and an exponential approach - and discuss their applicability taking into account two real-life applications, a high-bay rack feeder and an inverse pendulum.
\end{abstract}

Keywords: gain scheduling control design, cooperativity, interval enclosure techniques, LMIs

\section{Introduction}

The use of LMIs is common when dealing with robust control issues. Although formerly developed for purely linear systems, their use can be extended to nonlinear applications if they can be expressed in a quasi-linear state-space representation. Here, the state dependencies can be considered as uncertainties in the system's polytopic representation and/or input matrices which makes them further prone to be handled with LMIs. This quasi-linear state-space representation overapproximates

\footnotetext{
${ }^{a}$ Chair of Mechatronics, University of Rostock, Justus-von-Liebig Weg 6, D-18059 Rostock, Germany E-mail: \{Julia.Kersten, Andreas.Rauh, Harald. Aschemann\} Ouni-rostock.de
} 
the influence of the state dependencies. When dealing with intervals, overestimation is a big subject to current investigations. In [8], feedback gains were computed for an initial state interval with a subsequent verification step, in which it was examined whether the control is valid over a finitely long time horizon in terms of a verified stabilization of the system dynamics. Here, if the verification failed, the gain was adjusted after computing a bounding box of states that are reachable over the complete prediction window. However, the results showed that the verification step was difficult to perform with existing state-of-the-art approaches $[14,17]$ because of a high level of overestimation. This led to an increased interval width for the computed states in a short time. It became clear that this was mainly caused by the prediction step which was done by a Picard iteration with a subsequent tightening step evaluating a temporal Taylor series expansion of the initial value problem (IVP). To avoid this step, cooperativity has been considered, e.g. in [10] on the basis of findings in $[5,15,16]$. For an autonomous dynamic system

$$
\dot{\mathbf{x}}(t)=\mathbf{f}(\mathbf{x}(t)), \quad \mathbf{x} \in \mathbb{R}^{n},
$$

cooperativity is given as a sufficient condition when all off-diagonal elements $J_{i, j}$, $i, j \in\{1, \ldots, n\}, i \neq j$, of the corresponding Jacobian

$$
\mathbf{J}=\frac{\partial \mathbf{f}(\mathbf{x})}{\partial \mathbf{x}}
$$

are strictly non-negative according to

$$
J_{i, j} \geq 0, \quad i, j \in\{1, \ldots, n\}, \quad i \neq j .
$$

For such cases, it is guaranteed that state trajectories $\mathbf{x}(t)$ starting in the positive orthant

$$
\mathbb{R}_{+}^{n}=\left\{\mathbf{x} \in \mathbb{R}^{n} \mid x_{i} \geq 0, \quad \forall i \in\{1, \ldots, n\}\right\}
$$

stay in this positive orthant for all $t \geq 0$ because

$$
\dot{x}_{i}(t)=f_{i}\left(x_{1}, \ldots, x_{i-1}, 0, x_{i+1}, \ldots x_{n}\right) \geq 0
$$

holds for all components $i \in\{1, \ldots, n\}$ of the state vector as soon as the state $x_{i}$ reaches the value $x_{i}=0$. This property is often referred to as positivity of the system model (1) [7]. The computation of interval enclosures simplifies with this, because the worst-case bounds of uncertain systems can be computed following the element-wise inequalities (6) as two separate linear known systems and while assuring that all possible states lie within their solutions

$$
\mathbf{f}_{v}(\mathbf{v})=\dot{\mathbf{v}}(t) \leq \dot{\mathbf{x}}(t) \leq \dot{\mathbf{w}}(t)=\mathbf{f}_{w}(\mathbf{w}) \quad \text { with } \quad \mathbf{v} \leq \underline{\mathbf{x}} \text { and } \quad \mathbf{w} \geq \overline{\mathbf{x}} .
$$

There are system models that are naturally cooperative like in the fields of biological, chemical, and medical applications. However, other systems do not show this property, when derived by first-principle techniques. This holds for example for electrical, magnetic, and mechanical systems. Those systems can, as [10] shows, 
be transformed into a cooperative form. However, overestimation still occurs due to the associated similarity transformation, which has to be done in two directions (transforming the system into a cooperative coordinate system and then transferring the computed state enclosures back into the original form) leading to even larger intervals for some applications. Hence, if such direct state-space transformations are not effective enough, we make use of an exponential interval enclosure approach, developed in [19].

Section 2 presents two different approaches to combine the findings of a gain scheduling control and a verified state computation. A further look into the computation of the required interval enclosure is given in Section 3, describing both methods and discussing their general applicability. The next two sections will give application scenarios, with Section 4 regarding a parameter-dependent uncertainty in a high-bay rack feeder, while Section 5 shows the behavior of an inverse pendulum with uncertain initial conditions resulting in uncertainty in the states due to a quasi-linear state-space representation and the physically motivated dependencies between all states. Finally, Section 6 gives conclusions and an outlook on future work.

\section{Robust Gain Scheduling Control}

The basic idea for all approaches considered in this paper is to calculate controller gains for all reachable states to robustly control an uncertain system. Here, we want to guarantee asymptotic stability of the closed-loop system for all uncertain initial states described by the $n$-dimensional interval box

$$
\left[\mathbf{x}_{0}\right]=[\mathbf{x}](0)=\left[\begin{array}{c}
{\left[\underline{x}_{1}(0) ; \bar{x}_{1}(0)\right]} \\
\vdots \\
{\left[\underline{x}_{n}(0) ; \bar{x}_{n}(0)\right]}
\end{array}\right]
$$

where $\inf \left(\left[x_{i}\right]\right)=\underline{x}_{i}$ represents the infimum and $\sup \left(\left[x_{i}\right]\right)=\bar{x}_{i}$ the supremum of each vector component $\left[x_{i}\right]=\left[\underline{x}_{i} ; \bar{x}_{i}\right], i \in\{1, \ldots, n\}, \underline{x}_{i} \leq x_{i} \leq \bar{x}_{i}$. Two different possibilities of uncertainties of the system are considered. One is a parameter variability in a linear continuous-time system

$$
\begin{aligned}
& \dot{\mathbf{x}}=\mathbf{A}(\mathbf{p}) \cdot \mathbf{x}+\mathbf{B}(\mathbf{p}) \cdot \mathbf{u} \\
& \mathbf{y}=\mathbf{C} \cdot \mathbf{x}+\mathbf{D} \cdot \mathbf{u}
\end{aligned}
$$

and the other is an uncertainty due to state-dependency of a nonlinear continuoustime system in the form

$$
\dot{\mathbf{x}}=\mathbf{f}(\mathbf{x}, \mathbf{u})
$$

with the state vector $\mathbf{x} \in \mathbb{R}^{n}$, the parameter vector $\mathbf{p} \in \mathbb{R}^{n_{\mathrm{p}}}$, and the control vector $\mathbf{u} \in \mathbb{R}^{m}$. If Eq. (9) can be reformulated exactly in terms of the quasi-linear state-space representation

$$
\begin{aligned}
& \dot{\mathbf{x}}=\mathbf{A}(\mathbf{x}) \cdot \mathbf{x}+\mathbf{B}(\mathbf{x}) \cdot \mathbf{u} \\
& \mathbf{y}=\mathbf{C}(\mathbf{x}) \cdot \mathbf{x}+\mathbf{D}(\mathbf{x}) \cdot \mathbf{u}
\end{aligned}
$$


and assuming the desired operating state at $\mathbf{x}=\mathbf{x}_{\mathrm{s}}=\mathbf{0}$ for the steady-state input signal $\mathbf{u}=\mathbf{u}_{\mathrm{s}}=\mathbf{0}$, a feedback controller is parameterized according to

$$
\mathbf{u}=-\mathbf{K} \cdot \mathbf{x} \quad \text { or } \quad \mathbf{u}=-\mathbf{K}(\mathbf{x}) \cdot \mathbf{x} .
$$

Suitable control laws for the second option in Eq. (11) can be determined by means of extended linearization techniques [3]. Hence, both types of control laws can be handled equally when considering an uncertainty due to parameter variability or an uncertainty due to the state dependency if both controller gains are computed by means of an LMI approach. Note that this section is written to underline and discuss the general applicability of this approach. Hence, there will be notes and remarks which are not considered in the application scenarios but can be incorporated quickly and easily because of the chosen structure of the design method. Firstly, this section represents a state-of-the-art design which is then extended by two novel approaches to reduce conservativity if either fixed or piece-wise constant gains are determined for the control laws in (11).

\subsection{Robust LMI-Based Control Synthesis}

The advantage of this well known method lies in a direct robust design approach because the system model in Eq. (10) is overapproximated by a polytopic uncertainty representation, see [22], with parameter-dependent system and input matrices $\mathbf{A}(\mathbf{p})$ and $\mathbf{B}(\mathbf{p})$, which complies with Eq. (8) in a straightforward way. This model can be represented by the following convex combination of suitably chosen vertex matrices

$$
\mathcal{D}=\left\{[\mathbf{A}(\boldsymbol{\xi}), \mathbf{B}(\boldsymbol{\xi})] \mid[\mathbf{A}(\boldsymbol{\xi}), \mathbf{B}(\boldsymbol{\xi})]=\sum_{\nu=1}^{n_{\nu}} \xi_{v} \cdot\left[\mathbf{A}_{\nu}, \mathbf{B}_{\nu}\right] ; \sum_{\nu=1}^{n_{\nu}} \xi_{\nu}=1 ; \xi_{\nu} \geq 0\right\}
$$

with the help of the vector $\boldsymbol{\xi}=\left[\begin{array}{lll}\xi_{1} & \ldots & \xi_{\nu}\end{array}\right]^{T}$. Here, the vertex matrices are denoted by $\mathbf{A}_{\nu}=\mathbf{A}_{\nu}(\mathbf{p})$ and $\mathbf{B}_{\nu}=\mathbf{B}_{\nu}(\mathbf{p})$, where each of them depends in an affine way on the vector of independent parameters $\mathbf{p} \in \mathbb{R}^{n_{\mathrm{p}}}$ which are contained in the interval box

$$
[\mathbf{p}]=[\underline{\mathbf{p}} ; \overline{\mathbf{p}}]
$$

with the component-wise defined bounds $p_{i} \leq p_{i} \leq \bar{p}_{i}, i \in\left\{1, \ldots, n_{\mathrm{p}}\right\}$. Under this assumption of independent parameters, $n_{\nu}=2^{n_{\mathrm{p}}}$ vertex systems need to be taken into consideration for the robust control design, which result from an evaluation of $\mathbf{A}(\mathbf{p})$ and $\mathbf{B}(\mathbf{p})$ for each of the vertices

$$
\mathcal{P}=\left\{\left[\begin{array}{c}
\underline{p}_{1} \\
\underline{p}_{2} \\
\vdots \\
\underline{p}_{n_{\mathrm{p}}}
\end{array}\right],\left[\begin{array}{c}
\bar{p}_{1} \\
\underline{p}_{2} \\
\vdots \\
\underline{p}_{n_{\mathrm{p}}}
\end{array}\right], \ldots,\left[\begin{array}{c}
\bar{p}_{1} \\
\bar{p}_{2} \\
\vdots \\
\bar{p}_{n_{\mathrm{p}}}
\end{array}\right]\right\}=\left\{\mathbf{p}^{\langle 1\rangle}, \ldots, \mathbf{p}^{\left\langle n_{\nu}\right\rangle}\right\} .
$$

Now, LMIs give us many options to compute suitable controller gains based on our needs and desired specifications. The chosen implementation is done to maintain 
the highest possible generalization, so that it can easily be adjusted to different scenarios. For that, we make use of feasible regions of eigenvalues of the closedloop control system which are defined in terms of a so-called $\Gamma$-region ${ }^{1}$. These regions are introduced as

$$
\mathbf{F}_{\Gamma}(s)=\mathbf{D}_{0}+s \mathbf{D}_{1}+\bar{s} \mathbf{D}_{1}^{T}
$$

with the Laplace variable $s \in \mathbb{C}$ and its conjugate complex $\bar{s}$. Here, negative definiteness $\mathbf{F}_{\Gamma} \prec 0$ needs to be satisfied for all eigenvalues of the closed-loop system. To reformulate the inequality $\mathbf{F}_{\Gamma} \prec 0$ into an LMI, we consider the following: If all eigenvalues of a real-valued system matrix A lie within the interior of the region (15), a positive definite matrix $\mathbf{P}=\mathbf{P}^{T} \succ 0$ exists that fulfills the matrix inequality $[22]$

$$
\mathbf{D}_{0} \otimes \mathbf{P}+\mathbf{D}_{1} \otimes(\mathbf{A P})+\mathbf{D}_{1}^{T} \otimes(\mathbf{A P})^{T} \prec 0
$$

Here, the matrix $\mathbf{P}$ defines a Lyapunov function $V(\mathbf{x})=\frac{1}{2} \mathbf{x}^{T} \mathbf{P} \mathbf{x}>0$ for $\mathbf{x} \neq \mathbf{x}_{\mathrm{S}}=\mathbf{0}$ with which stability of the dynamic system $\dot{\mathbf{x}}=\mathbf{A x}$ can be proven. Moreover, the real-valued parameter matrices $\mathbf{D}_{0}=\mathbf{D}_{0}^{T}$ and $\mathbf{D}_{1}$ provide flexible possibilities as requested to define $\Gamma$-stability regions such as ellipses, hyperbolas, parabolas, cones, and strips in the complex plane [1].

An exemplary choice for an absolute stability margin $\gamma>0$ would lead to setting $D_{0}=2 \gamma$ and $D_{1}=1$ resulting in a design goal for the region $F_{\Gamma}=2 \gamma+s+\bar{s} \prec 0$ equivalent to a limitation of the eigenvalues' real parts by the inequality contraint $\Re\{s\}<-\gamma<0$. Note that pure Hurwitz stability is trivially included in this formulation by choosing $\gamma=0$. For the control design, the inequality (16) is reformulated according to

$$
\mathbf{D}_{0} \otimes \mathbf{Q}+\mathbf{D}_{1} \otimes\left(\mathbf{Q A}_{\nu}^{T}-\mathbf{Y}^{T} \mathbf{B}_{\nu}^{T}\right)+\mathbf{D}_{1}^{T} \otimes\left(\mathbf{A}_{\nu} \mathbf{Q}-\mathbf{B}_{\nu} \mathbf{Y}\right) \prec 0
$$

after a linearizing change of variables $\mathbf{Q}=\mathbf{P}^{-1}$ and $\mathbf{K}=\mathbf{Y} \mathbf{P}$. A joint solution $\mathbf{Q} \succ 0, \mathbf{Y}$ of the LMI (17) for each of the vertices $\nu \in\left\{1, \ldots, 2^{n_{\mathrm{p}}}\right\}$ is required to achieve robust stability for the uncertainty representation (12)-(14) with eigenvalues that are compatible with the domain $\mathbf{F}_{\Gamma} \prec 0$ defined in (15). This can be done numerically by using commonly known LMI solvers like SEDUMI [25] for YALMIP [12].

For the first scenario defined in Eq. (8), where the uncertainty lies within the parameters, this is basically the full approach. Here, there is only one controller gain for the whole range of parameters, see the first part of Eq. (11), and the only thing left for a simulation-based performance evaluation is to calculate worst-case bounds for all reachable states which will be addressed in Sec. 3 .

\subsection{Reducing Conservativity by Means of Gain Scheduling}

The novel approach is based on the idea that for systems with state dependencies, the initially chosen domain of all reachable states $[\mathbf{x}](t) \subseteq[\boldsymbol{\mathcal { X }}]$ for the complete

\footnotetext{
${ }^{1}$ Obviously, also optimality criteria such as robust $H_{2}$ and $H_{\infty}$ tasks can be taken into account by the same LMI-based design framework also considering output and state limitations, see $[4,6]$.
} 
operating time horizon $t \in\left[t_{0} ; t_{\mathrm{f}}\right]$, which is used for the LMI-based control parameterization, may (at least after a certain time span) be too conservative and, thus, turning the control parameterization unnecessarily restrictive. This conservativity can be reduced by gain scheduling controllers based on tight enclosures of the sets of reachable states Eq. (10). The approach is an extension on the idea of [8].

Here, the goal of the design procedure is to ensure asymptotic stability of the closed-loop system for all reachable states $\mathbf{x}(t) \in[\mathbf{x}](t)$ by a sequence of control matrices $\mathbf{K}_{k}, k \in\{0,1,2, \ldots\}$, where $T=t_{k+1}-t_{k}$ denotes a fixed sampling time. It was found, that after a certain time, the gains remain constant in the vicinity of the equilibrium. This is used in the presented paper for two different novel designs. Both approaches are based on the idea that we can predict guaranteed state enclosures for a robustly controlled state initialized by an interval. Hence, they basically aim at a reduction of the width of the reachable domains due to the control, reducing conservativity in the control strategy itself.

\subsubsection{Approach 1: Constant gain with robustness over the whole time horizon}

The first approach makes use of the information gained over the complete time horizon. For this, it firstly computes a controller gain for the interval domain of the initial states set by the user (usually the complete controllable domain), which includes all assumed reachable states $\left[\mathcal{X}^{(0)}\right]=[\check{\mathcal{X}}]$ for $t \in\left[t_{0} ; t_{\mathrm{f}}\right]$. This enclosure $[\check{\mathcal{X}}]$ is inflated to a rough outer enclosure of the desired operating domain, which is then subsequently tightened in an iterative procedure with $i \in \mathbb{N}$ to the actually reachable interval domains. For details about this procedure see also Sec. 3. Note that if $\left[\boldsymbol{\mathcal { X }}^{(i+1)}\right] \subseteq\left[\boldsymbol{\mathcal { X }}^{(i)}\right] \subseteq[\check{\mathcal{X}}]$ holds, the hull over all intervals of the state regarding the whole time horizon is set to the new less conservative interval bounds for the next iteration of the controller gains $\mathbf{K}^{(i+1)}$, see Fig. 1.

This is repeated as long as the interval diameters decrease, finalizing when the

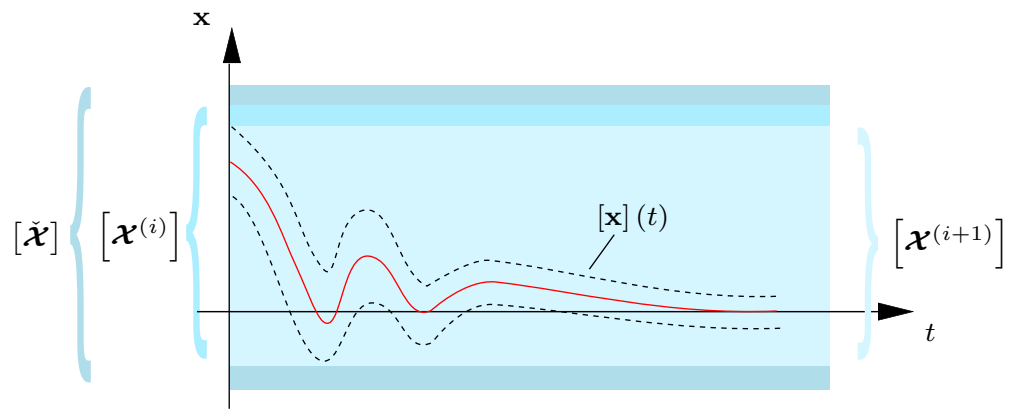

Figure 1: Illustration of the basic control approach for a scalar state variable. 
optimal solution with the smallest amount of conservativity regarding the controller gains is reached or if a final number of runs has been done as the yellow parts of the structure in Fig. 2 show.

Set $[\check{\mathcal{X}}]$ as the initial state domain $\left[\mathcal{X}^{(0)}\right], i:=0$

Compute a robust stabilizing controller gain $\mathbf{K}_{\text {ini }}$ (according to the specifications in Sec. 2.1) for the parameters $[\mathbf{p}]$ corresponding to the complete state interval which the system matrix depends on

Calculate the interval enclosures of reachable states for the complete time horizon $t=\left[t_{0} ; t_{\mathrm{f}}\right]$

Set the outer hull over all interval enclosures $\left[\boldsymbol{\mathcal { X }}^{(i)}\right] \underset{t \in\left[t_{0} ; t_{\mathrm{f}}\right]}{\bigcup}[\mathbf{x}]$ as the new state domain

Analyze the sequence of interval enclosures for optimal switching points and get the respective list of time steps $t_{\text {list }} \in\left\{t_{\zeta}\right\}, \zeta \in\left\{1, \ldots, \zeta_{\text {end }}\right\}$ at which the gain matrix $\mathbf{K}$ changes its value

End of new time vector has not been reached $t_{\mathrm{f}} \neq t_{\text {list }}\left(\zeta_{\text {end }}\right)$

Set simulation time to $t_{0}=t_{\text {list }}(\zeta)$ and $t_{\mathrm{f}}=t_{\text {list }}(\zeta+1)$

Final number of runs has not been reached $i \leq N \vee\left[\boldsymbol{\mathcal { X }}^{(i+1)}\right] \subseteq\left[\boldsymbol{\mathcal { X }}^{(i)}\right]$

Compute a robust stabilizing controller gain $\mathbf{K}=\mathbf{K}^{(i)}$ (according to the previous specifications) for the parameters [p] corresponding to the complete interval $[\mathcal{X}]:=\left[\mathcal{X}^{(i)}\right]$ state interval which the system matrix depends on ${ }^{2}$

Calculate the interval enclosures for the complete time horizon $t=\left[t_{0} ; t_{\mathrm{f}}\right]$

Set the hull over all interval enclosures $\left[\mathcal{X}^{(i)}\right]=\bigcup[\mathbf{x}](t)$ as the new state domain $t \in\left[t_{0} ; t_{\mathrm{f}}\right]$

Increment the run counter $i:=i+1$, output: gain $\mathbf{K}=\mathbf{K}^{(i)}$

Figure 2: Interval-based gain scheduling procedure for Approach 1 (yellow) and Approach 2 (all).

This approach, however, comes with a lengthy computation and can still be very conservative, which leads to the need of optimization in terms of a temporal series of controller gains, where the time intervals of piece-wise constant gains are not constant as stated above but rather result from an intelligent step size control.

\footnotetext{
${ }^{2}$ Note that for the first run, this step is omitted and $\mathbf{K}_{\text {ini }}$ is used as a controller gain.
} 


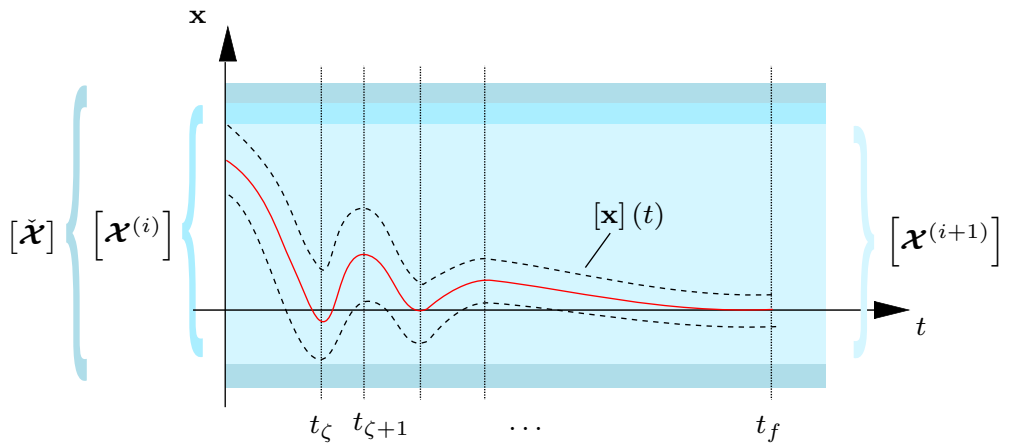

Figure 3: Illustration of the control approach for a scalar state variable.

\subsubsection{Approach 2: Gain scheduling design over temporal subslices}

Re-considering varying controller gains over the complete time horizon like in [8], the first approach is redesigned to divide the time horizon into shorter time slices while keeping everything else as it is. However, this would lead to an even longer computation time than before. An analysis of the previous approach shows that the time consuming computation comes from solving the LMIs for wide interval boxes. Hence, we now want to use various controller gains in the transient phase - wide interval boxes — while keeping constant ones for the later phase - tight interval boxes - , see Fig. 3. To achieve this, we make use of an initial run of the first approach to set all parameters and find a suitable division of the time horizon into time steps displaying the changes best. Now, the computation of the new boxes - the hull over all reachable states — is done for each of these steps analogously to the first approach like shown in Fig. 2. The very first run for each part of the time horizon, see also Fig. 9 and Eq. (56), — divided before — is done with the initially chosen, conservative interval domain of the complete time horizon. This is then gradually reduced with each iteration step re-scheduling the controller gains. After finding the optimum for the current time slice, the procedure is done for the next until the final time of the complete time horizon is reached.

\section{Interval Enclosure Techniques from the Perspective of Verified Gain Scheduling Control}

As mentioned in Sec. 2, we need to find interval enclosures to the IVP with uncertain initial conditions, see also [18], that allow for verifying the bounds that are included in the polytope (12). As described above, the computation of those interval enclosures is a difficult task to perform. In [8], it was done with a Picard iteration with a subsequent tightening step evaluating a temporal Taylor series expansion of the IVP. This resulted in wide interval bounds which had to be reduced 
in a second step by means of interval subdivisions and eliminations by different approaches such as computationally expensive stability considerations, see $[8,9]$. However, the resulting intervals were still able to become less conservative. Hence, the presented paper offers two different approaches.

\subsection{Transformation of the Closed-Loop System Model into a Cooperative Form}

For a first idea, we want to make use of the property of cooperativity as explained in Section 1. Since the system is already stabilized by methods given in Sec. 2, a suitable system representation is

$$
\dot{\mathbf{x}}=\mathbf{A}_{\mathrm{C}}(\mathbf{x}) \cdot \mathbf{x}
$$

with the controlled system matrix $\mathbf{A}_{\mathrm{C}}(\mathbf{x})=\mathbf{A}(\mathbf{x})-\mathbf{B}(\mathbf{x}) \cdot \mathbf{K}(\mathbf{x})$. Here, we assume that our system is not yet cooperative and, hence, needs to be transformed to calculate the worst-case bounds $[\mathbf{v}(t) ; \mathbf{w}(t)] \ni \mathbf{x}(t)$ and, therefore, the state interval enclosures. In case of cooperativity, this computation would be done by solving IVPs independently for the two decoupled bracketing systems [24]

$$
\inf \left(\mathbf{A}_{\mathrm{C}}([\mathcal{X}])\right) \cdot \mathbf{v}(t)=\dot{\mathbf{v}}(t) \leq \dot{\mathbf{x}}(t) \leq \dot{\mathbf{w}}(t)=\sup \left(\mathbf{A}_{\mathrm{C}}([\mathcal{X}])\right) \cdot \mathbf{w}(t)
$$

with the domain of reachable states $[\mathbf{x}](t) \subseteq[\boldsymbol{\mathcal { X }}]$. An approach to realize the transformation was presented in [10]. Here, we differentiate between systems with purely real eigenvalues, where one can use a time-invariant transformation and systems including conjugate-complex eigenvalues, where a time-varying transformation is necessary. For the given application scenario, we will restrict ourselves to the latter, considering disjoint pairs of conjugate-complex eigenvalues of the interval evaluation $\mathbf{A}_{\mathrm{C}}([\boldsymbol{\mathcal { X }}])$ of the closed-loop system matrix. In this case, in general only time-varying transformations into the form (19) are possible, see $[13,16]$. Here, we can map the uncertainty into the locations of the eigenvalues themselves, which is illustrated with an example for a system of order $n=2$ in Fig. 4.

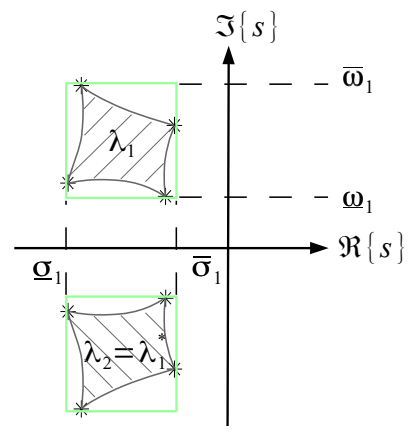

Figure 4: Possible locations of uncertain conjugate-complex eigenvalues. 
It becomes clear, that if the system matrices are evaluated for the whole range of uncertain parameters, this leads to a variability of the real and imaginary parts of conjugate-complex eigenvalues. Here, the position of the worst-case eigenvalues $i \in$ $\{1, \ldots, n\}$ for all possible vertex matrices, used before for a polytopic description of the uncertain system matrices, is marked by asterisk symbols. With those positions, we can define axes-parallel boxes as a convex outer interval hull describing the extremal real and imaginary parts $\left[\sigma_{i}\right]=\left[\underline{\sigma}_{i} ; \bar{\sigma}_{i}\right]$ and $\left[\omega_{i}\right]=\left[\underline{\omega}_{i} ; \bar{\omega}_{i}\right]$ of a conjugatecomplex eigenvalue pair. Note that when $n$ is the (even) number of states, assume without loss of generality $\tilde{n}=\frac{n}{2}$ guaranteed mutually disjoint conjugate-complex eigenvalue pairs. As it was shown in [18], also real eigenvalues can be included in this approach. There is a transformation matrix

$$
\tilde{\mathbf{T}}=\left[\tilde{\mathbf{T}}_{1}, \ldots, \tilde{\mathbf{T}}_{\tilde{n}}\right], \text { where } \tilde{\mathbf{T}}_{j} \in\left[\Re\left\{\left[\mathbf{v}_{j}\right]\right\}, \Im\left\{\left[\mathbf{v}_{j}\right]\right\}\right]
$$

with $j \in\{1, \ldots, \tilde{n}\}$, which consists of interval enclosures for the real and imaginary parts of the eigenvectors of the uncertain system. Those eigenvectors can be computed by means of the INTLAB routine verifyeig [21]. With that transformation, where $\mathbf{x}=\tilde{\mathbf{T}} \cdot \tilde{\mathbf{z}}$, a block diagonal transformed system matrix is formally obtained:

$$
\tilde{\mathbf{A}}=\operatorname{blkdiag}\left(\tilde{\mathbf{A}}_{1}, \ldots, \tilde{\mathbf{A}}_{\tilde{n}}\right) \quad \text { with } \quad \tilde{\mathbf{A}}_{j} \in\left[\begin{array}{cc}
{\left[\sigma_{j}\right]} & {\left[\omega_{j}\right]} \\
-\left[\omega_{j}\right] & {\left[\sigma_{j}\right]}
\end{array}\right]
$$

The respective time-varying transformation is done by

$$
\mathbf{z}=\mathbf{T}^{-1}(t) \cdot \tilde{\mathbf{z}} \quad \text { with } \quad \mathbf{T}^{-1}(t)=\operatorname{blkdiag}\left(\mathbf{T}_{1}^{-1}(t), \ldots, \mathbf{T}_{\tilde{n}}^{-1}(t)\right)=\mathbf{T}^{T}(t) .
$$

The orthogonal blocks

$$
\mathbf{T}_{j}=\left[\begin{array}{rr}
\cos \left(\left[\omega_{j}\right] t\right) & \sin \left(\left[\omega_{j}\right] t\right) \\
-\sin \left(\left[\omega_{j}\right] t\right) & \cos \left(\left[\omega_{j}\right] t\right)
\end{array}\right]
$$

for $j \in\{1, \ldots, \tilde{n}\}$ are evaluated for the outer interval enclosures of the imaginary parts of all eigenvalues which contain the exact angular frequencies according to the relation $\omega_{j}^{*} \in\left[\omega_{j}^{*}\right] \subseteq\left[\omega_{j}\right]$. Since the structure of the transformed system matrix (21) is known, the evaluation of this matrix is only necessary for i) proving that the transformation leads to a system matrix in Metzler form and ii) to determine enclosures $[\mathbf{z}](0)$ of the initial states as a function of $[\mathbf{x}](0)$ for verified simulations (and respectively for a backward transformation of the computed results for $t>0$ ). For i) we use symbolic formula manipulation on the basis of exact values $\omega_{j}^{*}$ considering the related differential equation (22) to calculate the state-space representation by

$$
\dot{\mathbf{z}}=\dot{\mathbf{T}}^{T}(t) \cdot \tilde{\mathbf{z}}+\mathbf{T}^{T}(t) \cdot \dot{\tilde{\mathbf{z}}}=\left[\left[\frac{\mathrm{d} \mathbf{T}^{T}(t)}{\mathrm{d} t}+\mathbf{T}^{T}(t) \tilde{\mathbf{A}}\right] \mathbf{T}(t)\right] \mathbf{z}=\mathbf{N} \cdot \mathbf{z} .
$$

Here, $\mathbf{N}$ is Metzler with real parts of the eigenvalues on the diagonal

$$
\mathbf{N}=\operatorname{blkdiag}\left(\sigma_{1} \mathbf{I}, \ldots, \sigma_{n} \mathbf{I}\right), \quad \mathbf{I}=\left[\begin{array}{ll}
1 & 0 \\
0 & 1
\end{array}\right],
$$


which can be shown by a symbolic simplification in terms of the exact values $\omega_{j}^{*}$ that are subsequently replaced by their conservative intervals $\left[\omega_{j}\right]$. As an analytical solution of Eq. (19), $\mathbf{z}_{i} \in e^{\left[\sigma_{i}\right] t} \cdot\left[\mathbf{z}_{i}\right](0)$ holds. Since $\mathbf{N}$ is evaluated for the eigenvalues, Hurwitz stability is verified for $\bar{\sigma}_{i}<0$. Extrema of the conjugate-complex eigenvalues are obtained by building the hull over their real parts $\left[\sigma_{j}\right]=\left[\min \left(\sigma_{j}\right) ; \max \left(\sigma_{j}\right)\right]$ as well as their imaginary parts $\left[\omega_{j}\right]=\left[\min \left(\omega_{j}\right) ; \max \left(\omega_{j}\right)\right]$, see [10]. Another variation of this approach is to omit the time-varying transformation by including the complex, uncertain eigenvector structure directly in the transformation matrix $\tilde{\mathbf{T}} \in \mathbb{C}^{n \times n}$. Here, Eq. (20) is reformulated into

$$
\tilde{\mathbf{T}}=\left[\tilde{\mathbf{T}}_{1}, \ldots, \tilde{\mathbf{T}}_{\tilde{n}}\right], \text { where } \tilde{\mathbf{T}}_{j}=\left[\left[\mathbf{v}_{j}\right],\left[\mathbf{v}_{j}^{*}\right]\right]
$$

leading to a complex block diagonal matrix $\tilde{\mathbf{A}}=\operatorname{blkdiag}\left(\tilde{\mathbf{A}}_{1}, \ldots, \tilde{\mathbf{A}}_{\tilde{n}}\right) \in \mathbb{C}^{n \times n}$ with

$$
\tilde{\mathbf{A}}_{j} \in\left[\tilde{\mathbf{A}}_{j}\right]=\left[\begin{array}{cc}
{\left[\sigma_{j}\right]+\jmath \cdot\left[\omega_{j}\right]} & 0 \\
0 & \left.\left[\sigma_{j}\right]-\jmath \cdot\left[\omega_{j}\right]\right]
\end{array}\right] .
$$

The symbolic computation of the interval enclosures for the states in the new complex-valued coordinate frame has the advantage that the diagonal structure of the matrix $\tilde{\mathbf{A}}$ leads to mutually decoupled state equations. Applying this, the interval $\left[\mathbf{x}_{0}\right]$ of initial states is transformed into the new coordinates $[\tilde{\mathbf{z}}]=[\tilde{\mathbf{T}}]^{-1} \cdot[\mathbf{x}]$, then the simulation is performed, and the results are, after that, transformed backward into $[\mathbf{x}]=[\tilde{\mathbf{T}}] \cdot[\tilde{\mathbf{z}}]$.

This approach, however, may be problematic in cases, where the evaluation of Eq. (20) leads to excessively wide bounds for $\tilde{\mathbf{T}} \in[\tilde{\mathbf{T}}]$ if the complete possible domain $[\boldsymbol{X}]$ is considered. These bounds are too wide, if the interval-valued inverse $[\tilde{\mathbf{T}}]^{-1}$ of $[\tilde{\mathbf{T}}]$ does not exist (possibly after using the union over submatrices $[\tilde{\mathbf{T}}]=\bigcup_{\mathcal{I}}\left[\tilde{\mathbf{T}}_{\mathcal{I}}\right]$ resulting from a domain splitting $[\boldsymbol{\mathcal { X }}]=\bigcup_{\mathcal{I}}\left[\boldsymbol{\mathcal { X }}_{\mathcal{I}}\right]$ to enclose the inverse $\tilde{\mathbf{T}}^{-1} \in \bigcup_{\mathcal{I}}\left[\tilde{\mathbf{T}}_{\mathcal{I}}{ }^{-1}\right]$ with less overestimation) or if it induces an excessive blow-up of the bounds due to the wrapping effect. Furthermore, this rather pessimistic — in terms of conservativity — calculation of $[\tilde{\mathbf{T}}]$ may also lead to a numerical instability in terms of a blow-up of interval enclosures. Controllability can only be lost, if $[\mathcal{X}]$ contains non-stabilizable points in the state space, which is detected by infeasible LMIs. For those cases, we make use of an alternative approach discussed in the next subsection.

\subsection{Exponential Interval Enclosure Technique}

In [11], an interface is derived between the exponential state enclosure technique presented in [19] and LMI-based approaches for robust control parameterization. Here, it is worth noticing, that the original exponential bounding approach in [19] was developed with no intent to combine it with any control design methodology. 
In the novel approach, an exponential interval enclosure for the true solution $\mathbf{x}^{*}(t)$ to an IVP with $\dot{\mathbf{x}}=\mathbf{f}(\mathbf{x})$ and $t_{0}=0$ is defined as

$$
\mathbf{x}^{*}(t) \in\left[\mathbf{x}_{e}\right](t):=\exp ([\mathbf{\Lambda}] \cdot t) \cdot\left[\mathbf{x}_{e}\right](0), \quad\left[\mathbf{x}_{e}\right](0)=\left[\mathbf{x}_{0}\right]
$$

with

$$
[\boldsymbol{\Lambda}]:=\operatorname{diag}\left[\lambda_{i}\right], i=\{1, \ldots, n\} .
$$

This correlates to the extended version of VALENCIA-IVP presented in $[19,20]$, where an iteration scheme was developed for the coefficients $\lambda_{i} \in \mathbb{R}$ introduced in (28) and (29) based on a Picard iteration

$$
\mathbf{x}^{*}(t) \in\left[\mathbf{x}_{e}\right]^{(\kappa+1)}:=\left[\mathbf{x}_{e}\right](0)+\int_{0}^{t} \mathbf{f}\left(\left[\mathbf{x}_{e}\right]^{(\kappa)}(s)\right) \mathrm{d} s .
$$

We now substitute the exponential state enclosures (28) for the exact solution in (30) and differentiate the resulting formula with respect to time leading to

$$
\dot{\mathbf{x}}^{*}(t) \in[\boldsymbol{\Lambda}]^{(\kappa+1)} \cdot \exp \left([\boldsymbol{\Lambda}]^{(\kappa+1)} \cdot t\right) \cdot\left[\mathbf{x}_{e}\right](0)=\mathbf{f}\left(\exp \left([\boldsymbol{\Lambda}]^{(\kappa)} \cdot t\right) \cdot\left[\mathbf{x}_{e}\right](0)\right)
$$

as a fixed-point iteration scheme. The evaluation of (31) is replaced by the expression

$$
\begin{aligned}
\dot{\mathbf{x}}^{*}([t]) & \in[\boldsymbol{\Lambda}]^{(\kappa+1)} \cdot \exp \left([\boldsymbol{\Lambda}]^{(\kappa+1)} \cdot[t]\right) \cdot\left[\mathbf{x}_{e}\right](0) \\
& =\mathbf{f}\left(\exp \left([\boldsymbol{\Lambda}]^{(\kappa)} \cdot[t]\right) \cdot\left[\mathbf{x}_{e}\right](0)\right)
\end{aligned}
$$

to account for the complete time interval $t \in[t]=[0 ; T]$. In the case of a converging iteration process given by Eqs. (31) and (32), the relations

$$
\left[\lambda_{i}\right]^{(\kappa+1)} \subseteq\left[\lambda_{i}\right]^{(\kappa)} \text { and } \quad[\boldsymbol{\Lambda}]^{(\kappa+1)} \subseteq[\boldsymbol{\Lambda}]^{(\kappa)}
$$

as well as

$$
\exp \left([\boldsymbol{\Lambda}]^{(\kappa+1)}[t]\right) \subseteq \exp \left([\boldsymbol{\Lambda}]^{(\kappa)} \cdot[t]\right)
$$

hold. Applying further reformulations of (32) according to $[19,20]$ as well as the convergence properties (33) and (34), the iteration formula

$$
\left[\lambda_{i}\right]^{(\kappa+1)}:=\frac{f_{i}\left(\exp \left([\boldsymbol{\Lambda}]^{(\kappa)} \cdot[t]\right) \cdot\left[\mathbf{x}_{e}\right](0)\right)}{\exp \left(\left[\lambda_{i}\right]^{(\kappa)} \cdot[t]\right) \cdot\left[x_{e, i}\right](0)}, i \in\{1, \ldots, n\},
$$

is obtained for the interval parameter $\left[\lambda_{i}\right]$ of the desired state enclosure. Note that the value 0 has to be excluded from the state enclosure or handled in another way in the computation of Eq. (35), because the involved division only holds for $0 \notin\left[x_{e, i}\right]$. 
As the final result of the iteration (35), the solution of all reachable states at $t=T$ (i.e., at the end of the considered integration horizon) is given by

$$
\mathbf{x}^{*}(t) \in\left[\mathbf{x}_{e}\right](t):=\exp ([\mathbf{\Lambda}] \cdot T) \cdot\left[\mathbf{x}_{e}\right](0),
$$

where $[\boldsymbol{\Lambda}]$ is composed of the result obtained in the final iteration step.

Since we work with systems with conjugate complex eigenvalues, the decoupling of the state equations - as one of the requirements for a maximization of efficiency of the exponential enclosure approach along with a domination by asymptotically stable, linear dynamics [19] — is not possible for those systems if only a transformation into real Jordan canonical form is performed. A possible solution proposed by the authors maintains the decoupling properties approximately by exploiting a transformation of a point-valued realization embedded in the uncertain system model into the complex Jordan canonical form. Both, linear and nonlinear, systems with uncertain parameters can be decoupled approximately if the matrix of the eigenvectors of the system's Jacobian, evaluated at the corresponding interval midpoints is used to perform the coordinate transformation before application of Eq. (35). After the computation of the complex-valued state enclosures, the results are transformed back into the original coordinates. Further details can be found in $[11,18-20]$. The basic simulation routine according to Eq. (28)-(36) was published in $[19,20]$ with a fixed, time- and state-independent integration step size. For the considered application scenario, it was extended by a simple step-size control strategy in [11] to guarantee numerical efficiency of the exponential enclosure technique. The step-size control strategy determines the most appropriate step size $T=T_{k}$ according to

$$
T_{k}=\max \left\{t_{\min }, \frac{1}{10} \cdot \min _{i \in \mathcal{I}^{*}}\left\{\inf \left\{\frac{-1}{\Re\left(\left[\lambda_{i}\right]\right)}\right\}\right\}\right\},
$$

for $k \in\{2,3, \ldots\}$, where $\mathcal{I}^{*}$ denotes the index set for all states and respective parameter enclosures $i \in\{1, \ldots, n\}$ for which the relation $0 \notin\left[\lambda_{i}\right]$ holds. In (37), the value $T_{1}$ is set to $T_{1}=t_{\text {min }}$. Furthermore, the generally complex-valued enclosures $\left[\lambda_{i}\right]$ in (37) are set to the results of the iteration that was performed during the evaluation of the state enclosures according to (35) for the last temporal discretization slice $[t]=\left[0 ; T_{k-1}\right]$, where the initial point of time of each slice is shifted to zero without loss of generality for time-invariant ODEs, cf. (35). Hence, this procedure for the adaptation of the integration step size $T$ by the sequence $\left\{T_{1}, T_{2}, \ldots\right\}$ results in the computation of state enclosures at the points of time $t=t_{k}$ with $t_{k}=\sum_{j=1}^{k} T_{j}$ instead of an equidistant grid $t_{k}=k \cdot T$ that would have to be adjusted to the fastest time constant for the complete simulation time horizon. 


\section{Application Scenario 1: High-Bay Rack Feeder}

The first considered scenario is a high-bay rack feeder as presented in Fig. 5. Since

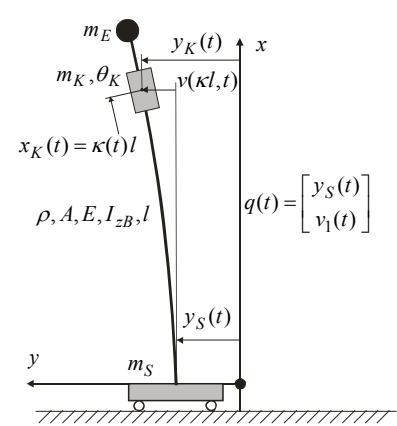

Figure 5: Mechanical model of the stacker crane.

this is an existing test-rig at the Chair of Mechatronics at the University of Rostock, previous work (cf. $[2,19])$ has already developed a control-oriented modeling as an elastic multibody system. This multibody model consists of three rigid bodies, namely a carriage with the mass $m_{S}$, a cage movable on a vertical double beam structure with mass $m_{K}$ and the mass moment of inertia $\theta_{K}$, as well as an end mass $m_{E}$ at the tip of the beam. A Bernoulli beam defines the elastic component with the density $\rho$, the cross sectional area $A$, Young's modulus $E$, the second moment of area $I_{z B}$, and the length $l$. A dimensionless system parameter

$$
\kappa(t)=\frac{x_{K}(t)}{l}
$$

denotes the time-varying vertical position $x_{K}(t)$ of the cage on the beam and is chosen as the uncertain parameter of the system. A Ritz ansatz

$$
\begin{gathered}
v(x, t)=\left[\begin{array}{ll}
\bar{v}_{1}(x) & \overline{\bar{v}}_{2}(x)
\end{array}\right]\left[\begin{array}{l}
v_{1}(t) \\
v_{2}(t)
\end{array}\right] \text { with } \\
\overline{\bar{v}}_{1}(x)=\frac{3}{2}\left(\frac{x}{l}\right)^{2}-\frac{1}{2}\left(\frac{x}{l}\right)^{3} \text { and } \quad \overline{\bar{v}}_{2}(x)=\left(\frac{x}{l}\right)^{2},
\end{gathered}
$$

is employed to describe the bending deflection of the beam structure by the corresponding elastic degrees of freedom, taking into account the first and the second bending mode. By applying Lagrange's equations of second kind, cf. [23], a secondorder ODE model

$$
\mathbf{M} \ddot{\mathbf{q}}(t)+\mathbf{D} \dot{\mathbf{q}}(t)+\mathbf{K q}(t)=\mathbf{h} \cdot\left(F_{S M}(t)-F_{S R}(t)\right)
$$

can be derived with the vector of generalized coordinates

$$
\mathbf{q}(t)=\left[\begin{array}{lll}
y_{S}(t) & v_{1}(t) & v_{2}(t)
\end{array}\right]^{T} .
$$


Assuming an underlying velocity control operating on the electric drive for the carriage with mass $m_{S}$, the resulting dynamics for $\ddot{y}_{S}$ can be replaced by a firstorder lag system with the time constant $T_{1 y}$ according to

$$
T_{1 y} \ddot{y}_{S}(t)+\dot{y}_{S}(t)=v_{S}(t)-v_{S 0}
$$

with the usually negligibly small input disturbance $v_{S 0}$. Substituting the ODE (43) into (41) leads to the equations of motion that are finally given by

$$
\ddot{\mathbf{q}}=-\mathbf{M}_{y}^{-1} \mathbf{K}_{y} \mathbf{q}-\mathbf{M}_{y}^{-1} \mathbf{D}_{y} \dot{\mathbf{q}}+\mathbf{M}_{y}^{-1} \mathbf{h}_{y} v_{S}
$$

with the carriage velocity $v_{S}$ as the new control input $u_{y}$. Here, the modified mass matrix

$$
\mathbf{M}_{y}(\kappa)=\left[\begin{array}{ccc}
T_{1 y} & 0 & 0 \\
m_{12} & m_{22} & m_{23} \\
m_{13} & m_{23} & m_{33}
\end{array}\right]
$$

is given with

$$
\begin{aligned}
& m_{12}=\frac{3}{8} \rho A l+\frac{m_{K} \kappa^{2}}{2}(3-\kappa)+m_{E} \\
& m_{13}=\frac{1}{3} \rho A l+m_{K} \cdot \kappa^{2}+m_{E} \\
& m_{22}=\frac{33}{140} \rho A l+\frac{6 \rho I_{z B}}{5 l}+\frac{m_{K} \kappa^{4}}{4}(3-\kappa)^{2}+\frac{9 \theta_{K} \kappa^{2}}{4 l^{2}}(2-\kappa)^{2}+m_{E} \\
& m_{23}=\frac{13}{60} \rho A l+\frac{5 \rho I_{z B}}{4 l}+\frac{m_{K} \kappa^{4}}{2}(3-\kappa)+\frac{3 \theta_{K} \kappa^{2}}{l^{2}}(2-\kappa)+m_{E} \\
& m_{33}=\frac{1}{5} \rho A l+\frac{4 \rho I_{z B}}{3 l}+m_{K} \kappa^{4}+\frac{4 \theta_{K} \kappa^{2}}{l^{2}}+m_{E} .
\end{aligned}
$$

The damping and the stiffness matrices correspond to

$$
\mathbf{D}_{y}=\left[\begin{array}{ccc}
1 & 0 & 0 \\
0 & \frac{3 k_{d} E I_{z B}}{l^{3}} & \frac{3 k_{d} E I_{z B}}{l^{3}} \\
0 & \frac{3 k_{d} E I_{z B}}{l^{3}} & \frac{4 k_{d} E I_{z B}}{l^{3}}
\end{array}\right] \quad \text { and } \quad \mathbf{K}_{y}(\kappa)=\left[\begin{array}{ccc}
0 & 0 & 0 \\
0 & k_{22} & k_{23} \\
0 & k_{23} & k_{33}
\end{array}\right]
$$

with

$$
\begin{aligned}
& k_{22}=\frac{3 E I_{z B}}{l^{3}}-\frac{3}{8} \rho A g-\frac{3 m_{K} g \kappa^{3}}{l}\left(1+\frac{3 \kappa^{2}}{20}-\frac{3 \kappa}{4}\right)-\frac{6 m_{E} g}{5 l} \\
& k_{23}=\frac{3 E I_{z B}}{l^{3}}-\frac{7}{20} \rho A g+\frac{m_{K} g \kappa^{3}}{l}\left(\frac{3 \kappa}{4}-2\right)-\frac{5 m_{E} g}{4 l} \\
& k_{33}=\frac{4 E I_{z B}}{l^{3}}-\frac{1}{3} \rho A g-\frac{4 m_{K} g \kappa^{3}}{3 l}-\frac{4 m_{E} g}{3 l} .
\end{aligned}
$$

Finally, the input vector of generalized forces is represented by $\mathbf{h}=\left[\begin{array}{ccc}1 & 0 & 0\end{array}\right]^{T}$. For the purpose of a feedback control design, the system is transformed into its 
state-space representation

$$
\dot{\mathbf{x}}_{y}=\left[\begin{array}{cc}
\mathbf{0} & \mathbf{I} \\
-\mathbf{M}_{y}^{-1} \mathbf{K}_{y} & -\mathbf{M}_{y}^{-1} \mathbf{D}_{y}
\end{array}\right]\left[\begin{array}{c}
\mathbf{q} \\
\dot{\mathbf{q}}
\end{array}\right]+\left[\begin{array}{c}
\mathbf{0} \\
\mathbf{M}_{y}^{-1} \mathbf{h}_{y}
\end{array}\right] v_{S}
$$

with a parameter uncertainty in $\mathbf{M}_{y}$ and $\mathbf{K}_{y}$ due to their dependency on $\kappa$. For the simulation, the parameter domain to be considered shall be $[\kappa]=[0.35 ; 0.5]$. As discussed previously, a robust LMI-based controller $v_{S}=-\mathbf{K} \cdot\left[\begin{array}{ll}\mathbf{q} & \dot{\mathbf{q}}\end{array}\right]^{T}$ is designed according to Sec. 2.1, numerical values can be found in the Appendix. The resulting interval enclosures are computed by using a transformation into cooperative form. Fig. 6 shows the result for the carriage position with an initial uncertainty of $y_{S}=$ $[0.1 ; 0.3] \mathrm{m}$.

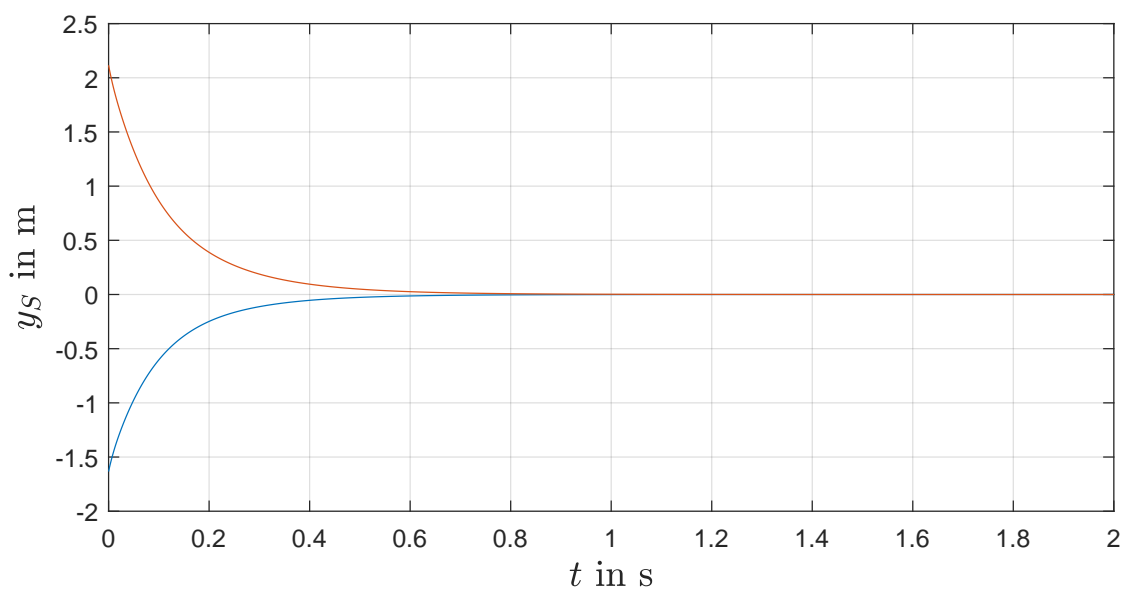

Figure 6: Upper and lower bound of the interval for the carriage position $y_{S}$ for all $t \in\left[0 ; t_{\mathrm{f}}\right]$

One can see that the controller works fast and efficiently in reaching the desired operating point $y_{S 0}=0 \mathrm{~m}$. Due to the asymptotic stability, both decoupled bounds converge to said stationary operating point. If a feedforward control is implemented, the asymptotic stability would be reduced to input to state stability (ISS) due to bounded uncertainty in the mass matrix $\mathbf{M}_{y}$, typically imposing uncertainty in the stationary system gain. However, in the depicted simulation, there is a deviation from the true ${ }^{3}$ state in the starting phase. This happens due to the overestimated transformations according to Eqs. (20)-(25) and the resulting overestimation of the complex eigenvalues. A possible solution could be to interface this method with the approaches from [19] e.g. applying complex-valued Jordan canonical forms. It becomes clear that this problem only occurs in a short time interval of approximately $1.9 \mathrm{~s}$ till realistic values - regarding the test rig - are reached in the interval-based simulation. We further compare the response times

\footnotetext{
${ }^{3}$ The rack feeder in question is a small scale model on a $1.5 \mathrm{~m}$ test rig.
} 
of our method with a grid-based simulation for the parameter $\kappa$ in Table 1 . For the gridding method, we simulated our original system with ten equally spaced grid-points included in the interval of $\kappa$.

Table 1: Comparison of response times for the interval computation vs. a parameter gridding.

\begin{tabular}{|c|c|c|c|}
\hline & \multicolumn{3}{|c|}{ deviation } \\
computation via & $\leq 0.1 \mathrm{~m}$ & $\leq 0.05 \mathrm{~m}$ & $\leq 0.01 \mathrm{~m}$ \\
\hline \hline gridding & $0.15 \mathrm{~s}$ & $0.25 \mathrm{~s}$ & $0.48 \mathrm{~s}$ \\
interval & $0.38 \mathrm{~s}$ & $0.51 \mathrm{~s}$ & $0.75 \mathrm{~s}$ \\
\hline
\end{tabular}

Here, the times show when the specified deviation to the stationary point $y_{S}=0$ is reached in each approach. The problem in gridding is to find suitable values to include all worst cases. In contrast to that, the interval method definitely includes such cases as it is oriented on the slowest time constant of the overall system. With that in mind, we can show that except for the short starting phase, the response time of the controlled system can be estimated well by means of the interval procedure.

\section{Application Scenario 2: Inverse Pendulum}

The second benchmark application is the stabilization of an inverted pendulum in its upright position. The control task is schematically represented in Fig. 7.

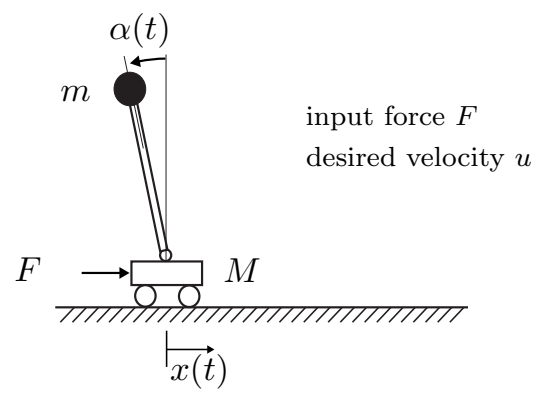

Figure 7: Control of an inverted pendulum on a moving carriage.

According to this, a pendulum of length $a=0.2 \mathrm{~m}$ is mounted at the horizontal foot-point position $x$ on a carriage of the mass $M$ moving along a track. The angle $\alpha$ denotes the deflection of the pendulum from its unstable upright equilibrium. In good accuracy, the pendulum can be described by a massless rod, while its mass $m$ is located in the tip of the pendulum. Under these assumptions, the system can be described by the following nonlinear autonomous second-order differential equation

$$
m a^{2} \cdot \ddot{\alpha}-m a \cdot \cos (\alpha) \cdot \ddot{x}-m g a \cdot \sin (\alpha)=0,
$$


with the gravitational acceleration $g=9.81 \frac{\mathrm{m}}{\mathrm{s}^{2}}$, and

$$
(M+m) \cdot \ddot{x}-m a \cdot \cos (\alpha) \cdot \ddot{\alpha}+m a \cdot \sin (\alpha) \cdot \dot{\alpha}=F,
$$

where $F$ is the actuation force applied to the carriage into the positive direction of motion $x$. Assuming an underlying velocity control for the carriage in the form of a first-order lag behavior with the time constant $T_{1}=0.05 \mathrm{~s}$, the expression (51) can be replaced by

$$
T_{1} \cdot \ddot{x}+\dot{x}=u,
$$

where $u$ and $\dot{x}$ represent the desired and actual carriage velocities, respectively. Hence, the overall system dynamics can be reformulated into a quasi-linear statespace representation

$$
\begin{aligned}
& \dot{\mathbf{x}}=\left[\begin{array}{cccc}
0 & 0 & 1 & 0 \\
0 & 0 & 0 & 1 \\
\frac{g \cdot \operatorname{si}(\alpha)}{a} & 0 & 0 & -\frac{\cos (\alpha)}{T_{1} a} \\
0 & 0 & 0 & -\frac{1}{T_{1}}
\end{array}\right] \mathbf{x}+\left[\begin{array}{c}
0 \\
0 \\
\frac{\cos (\alpha)}{T_{1} a} \\
\frac{1}{T_{1}}
\end{array}\right] u, \\
& y=\left[\begin{array}{llll}
-a \cdot \operatorname{si}(\alpha) & 1 & 0 & 0
\end{array}\right] \mathbf{x}, \quad \operatorname{si}(\alpha)=\frac{\sin (\alpha)}{\alpha} \text {, }
\end{aligned}
$$

with the state vector $\mathbf{x}=\left[\begin{array}{llll}\alpha & x & \dot{\alpha} & \dot{x}\end{array}\right]^{T}$ and the system input $u$. The initial state interval $[\mathbf{x}](0)$ is assumed to be represented by an uncertainty in the pendulum angle according to $[\mathbf{x}](0)=[[\alpha](0) \quad 0 \quad 0 \quad 0]^{T}$, where $[\alpha](0)=[\underline{\alpha}(0) ; \bar{\alpha}(0)]$.

To obtain a polytopic uncertainty representation (12) from the quasi-linear statespace representation in terms of a convex combination of extremal system models for the LMI-based control design, the matrix entries depending on the pendulum angle $\alpha$ are replaced by the two independent parameters

$$
p_{1}=\frac{g \cdot \operatorname{si}(\alpha)}{a} \quad \text { and } \quad p_{2}=\frac{\cos (\alpha)}{T_{1} \cdot a},
$$

so that the parameter-dependent system matrix and input vector

$$
\mathbf{A}(\mathbf{p})=\left[\begin{array}{cccc}
0 & 0 & 1 & 0 \\
0 & 0 & 0 & 1 \\
p_{1} & 0 & 0 & -p_{2} \\
0 & 0 & 0 & -\frac{1}{T_{1}}
\end{array}\right] \quad \text { and } \quad \mathbf{b}(\mathbf{p})=\left[\begin{array}{c}
0 \\
0 \\
p_{2} \\
\frac{1}{T_{1}}
\end{array}\right],
$$

respectively, are obtained. This model is used to simulate both gain scheduling approaches, see Section 2.2, for the uncertain system due to the state-dependency. The application scenario at hand is an example where a transformation into a cooperative form is not possible due to too wide intervals and, hence, a non-invertible transformation matrix occurs. Therefore, the exponential enclosure technique is used to determine interval enclosures. At first, Approach 1 is investigated for the control design (11) (Sec. 2). 


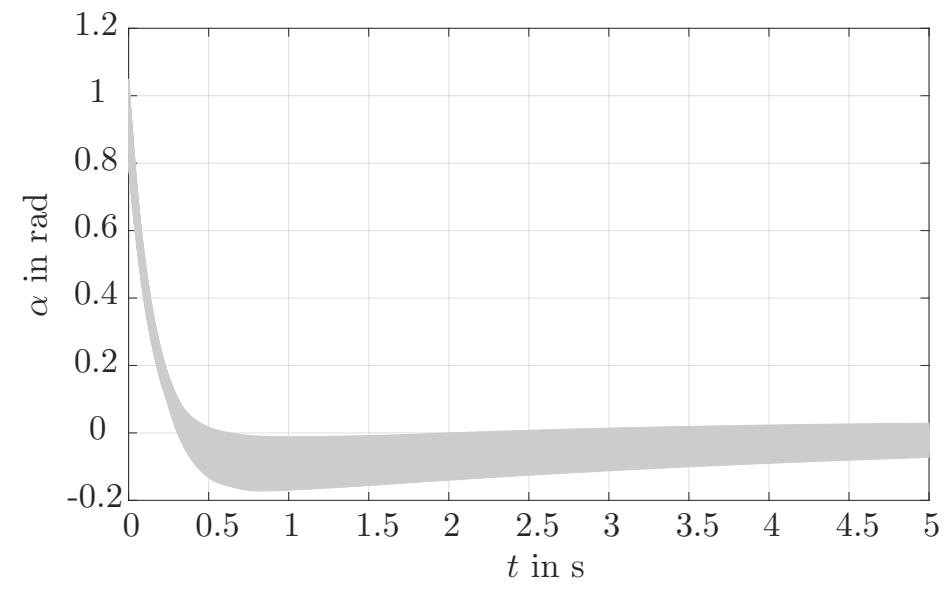

Figure 8: Interval enclosure for the pendulum angle $\alpha$ for all $t \in\left[0 ; t_{\mathrm{f}}\right]$.

Fig. 8 shows the resulting interval enclosure for the simulated model, exemplary for the third run $(N=3)$ with the initial state domain $\left[\mathcal{X}^{(0)}\right]=\left[-\frac{\pi}{2}+\epsilon ; \frac{\pi}{2}-\epsilon\right]$. Here, one can clearly see that the controller stabilizes the system dynamics successfully in its operating point. Furthermore, the interval diameter is decreasing once the operating point has been reached. Numerical results for the sequence of controller gains and the hull over the angle intervals for $t \in\left[0 ; t_{\mathrm{f}}\right]$ are given in Table 2. The first run shows the initial simulation over the complete time horizon with the most conservative controller gains resulting from the initial interval. In the second run, we see that the controller gains decrease due to a less conservative interval box describing the interval enclosure over the complete time horizon. This is repeated in the third run. The simulation shows, that a further reduction is not possible and, hence, the optimal solution has been found.

Table 2: Simulation results for Approach 1: Controller gains and enclosure of the first state variable, where underlined digits highlight the values identical between two successive iterations.

\begin{tabular}{|c|l|l|l|l|l|l|}
\hline run $i$ & \multicolumn{3}{|c|}{$K$} & \multicolumn{2}{|c|}{$\left[\mathcal{X}^{(i)}\right]_{1}$} \\
& $k_{1}$ & $k_{2}$ & $k_{3}$ & $k_{4}$ & inf & sup \\
\hline \hline 1 & 96.54707 & 15.3023677 & -0.472897180 & -4.99555847 & $\underline{-0.1521028}$ & $\underline{1.0481524366}$ \\
2 & 94.68120 & 15.0066626 & -0.463765567 & -4.89774783 & $\underline{-0.1721871}$ & $\underline{1.0481472189}$ \\
3 & 94.68119 & 15.0066613 & -0.463765526 & -4.89774740 & -0.1721838 & 1.0481472163 \\
\hline
\end{tabular}

For the application scenario at hand, Approach 2 described in Sec. 2.2.2 does not provide an improved control accuracy. However, we gain the information, when certain components of the state vector are mapped into themselves, see Fig. 9 as 
a simulation-based verification of the contraction property towards the asymptotically stable equilibrium. Here, the interval boxes are depicted exemplarily for $\alpha$

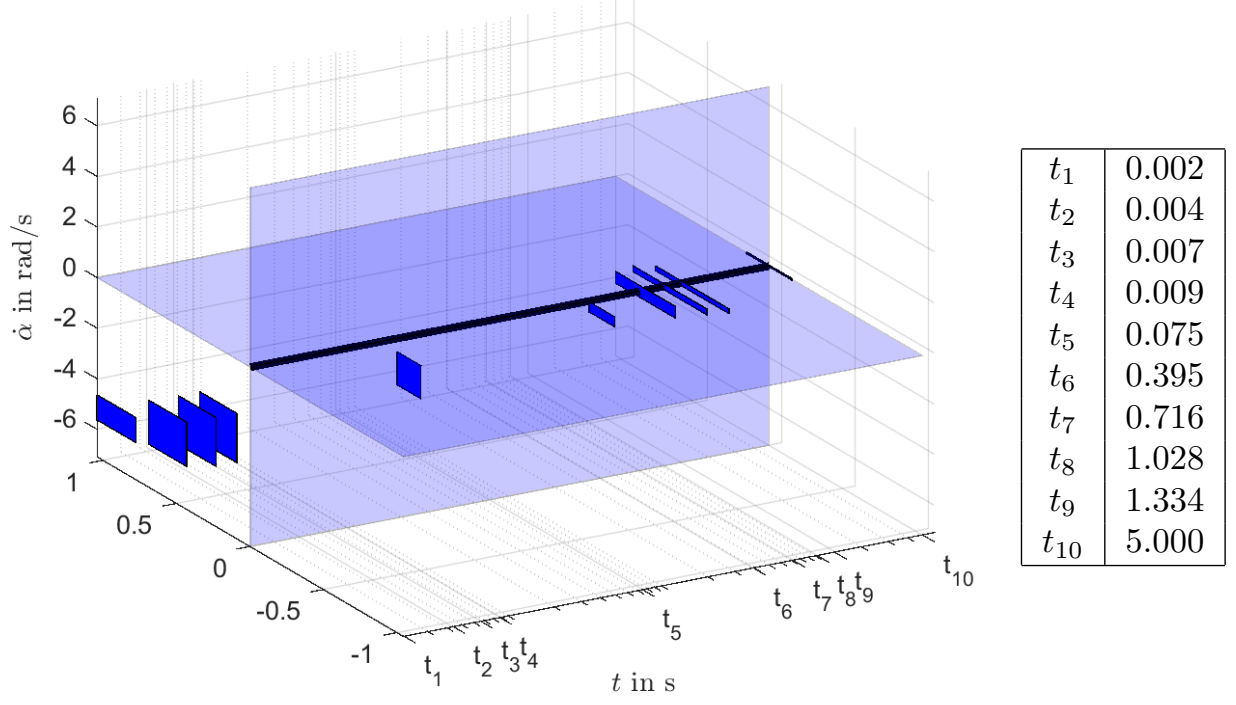

$\alpha$ in $\operatorname{rad}$

Figure 9: Approach 2: Intervalboxes for $\alpha$ and $\dot{\alpha}$ for each predefined time step $t_{\zeta}$ (depicted on a logarithmic temporal axis).

and $\dot{\alpha}$ over the time horizon $t_{\mathrm{f}}$. Note, that due to the step-size control strategy, we only have comparable enclosures for the fixed time steps $t_{\zeta} \in\left\{0, \tau_{i^{*}}, \tau_{2 i^{*}}, \ldots, t_{\mathrm{f}}\right\}$, which were already introduced in 2 as the points of time where the controller gains change, are calculated on the basis of the step size $T_{K}$ from Eq. (37) by

$$
i^{*}=\left\lceil\frac{L}{\zeta_{\text {end }}}\right\rceil,
$$

where $L$ is the number of all discretization steps from before, see the table in Fig. 9. Those time steps $t_{\zeta}$ are featured logarithmically, because - as predicted much denser time steps where needed for the beginning phase where the dynamics is more stiff than close to the steady state. Moreover, it must be noted, that for small time steps, the exponential enclosure is unnecessary conservative due to the involved transformation of the model into an approximately decoupled form. However, the stabilizing behavior is clearly visible and once the system gets close to the equilibrium, interval enclosures are getting tighter by exploiting the step size selection according to Eq. (37). 


\section{Conclusions and Future Work}

Uncertain systems were investigated in forms of descriptions with parameter uncertainty as well as a nonlinear models, where the uncertainty was given by the state-dependency of the system matrix. Two gain scheduling approaches were proposed for a feedback control synthesis and implementation, with the aim of improving control accuracy especially for the nonlinear system and its state dependency. Hence, in order to apply gain scheduling, a reliable verified enclosure technique for the states needs to be found, especially when using uncertainty due to state dependency since it directly influences the robust controller gains. Here, two methods were discussed, a transformation into cooperative form and an exponential interval enclosure technique. Both were investigated for applicability and general instructions for their respective choice were given. Successful simulations for reallife application scenarios verify the approaches. Future work will concentrate on applying the presented robust control strategy to real-life scenarios in electrical circuits as well as mechanical systems.

\section{A Numerical Values of Interval Matrices}

\section{A.1 Application Scenario 1}

Concerning the first application scenario (control of the high-bay rack feeder), the numerical values for the controlled system (49) are

$$
\begin{aligned}
\mathbf{A}_{\mathrm{C}} & =(\mathbf{A}-\mathbf{B K}) \\
& =\left[\begin{array}{cccccc}
0 & 0 & 0 & 1 & 0 & 0 \\
0 & 0 & 0 & 0 & 1 & 0 \\
0 & 0 & 0 & 0 & 0 & 1 \\
-1.44 \cdot 10^{3} & 8.15 \cdot 10^{3} & 9.43 \cdot 10^{3} & -0.28 \cdot 10^{3} & 0.03 \cdot 10^{3} & -0.09 \cdot 10^{3} \\
{\left[a_{51}\right]} & {\left[a_{52}\right]} & {\left[a_{53}\right]} & {\left[a_{54}\right]} & {\left[a_{55}\right]} & {\left[a_{56}\right]} \\
{\left[a_{61}\right]} & {\left[a_{62}\right]} & {\left[a_{63}\right]} & {\left[a_{64}\right]} & {\left[a_{65}\right]} & {\left[a_{66}\right]}
\end{array}\right]
\end{aligned}
$$

with

$$
\begin{array}{lll}
{\left[a_{51}\right]=[0.168 ; 0.207] \cdot 10^{5}} & {\left[a_{52}\right]=[-1.048 ;-0.840] \cdot 10^{5}} \\
{\left[a_{53}\right]=[0.121 ; 0.254] \cdot 10^{5}} & {\left[a_{54}\right]=[0.032 ; 0.040] \cdot 10^{5}} \\
{\left[a_{55}\right]=[-0.004 ;-0.003] \cdot 10^{5}} & {\left[a_{56}\right]=[0.012 ; 0.016] \cdot 10^{5}}
\end{array}
$$

and

$$
\begin{array}{lll}
{\left[a_{61}\right]=[-0.194 ;-0.154] \cdot 10^{5}} & {\left[a_{62}\right]=[0.745 ; 0.957] \cdot 10^{5}} \\
{\left[a_{63}\right]=[-0.401 ;-0.270] \cdot 10^{5}} & {\left[a_{64}\right]=[-0.038 ;-0.029] \cdot 10^{5}} \\
{\left[a_{65}\right]=[0.003 ; 0.005] \cdot 10^{5}} & {\left[a_{66}\right]=[-0.015 ;-0.011] \cdot 10^{5} .}
\end{array}
$$


This is transformed into

$$
\tilde{\mathbf{A}}_{\mathrm{C}}=\left[\begin{array}{cccccc}
{\left[\tilde{a}_{11}\right]} & 0 & 0 & 0 & 0 & 0 \\
0 & {\left[\tilde{a}_{22}\right]} & 0 & 0 & 0 & 0 \\
0 & 0 & {\left[\tilde{a}_{33}\right]} & 0 & 0 & 0 \\
0 & 0 & 0 & {\left[\tilde{a}_{44}\right]} & 0 & 0 \\
0 & 0 & 0 & 0 & {\left[\tilde{a}_{55}\right]} & 0 \\
0 & 0 & 0 & 0 & 0 & {\left[\tilde{a}_{66}\right]}
\end{array}\right] \cdot 10^{3}
$$

with the closed-loop matrix entries

$$
\begin{array}{llrl}
{\left[\tilde{a}_{11}\right]} & =\langle-1.90+0 i, 0.181\rangle & & {\left[\tilde{a}_{22}\right]=\langle-0.021+0.15 i, 0.004\rangle} \\
{\left[\tilde{a}_{33}\right]=\langle-0.02-0.14 i, 0.004\rangle} & & {\left[\tilde{a}_{44}\right]=\langle-0.006+0 i, 0.001\rangle} \\
{\left[\tilde{a}_{55}\right]=\langle-0.017+0.03 i, 0.005\rangle} & & {\left[\tilde{a}_{66}\right]=\langle-0.017-0.03 i, 0.005\rangle}
\end{array}
$$

in the midpoint-radius-form $[a]=\langle$ midpoint, radius $\rangle$, where the intervals are rounded in outward direction to the number of displayed digits.

\section{A.2 Application Scenario 2}

The second application scenario (control of the inverse pendulum) is given by Eq. (53) with

$$
\mathbf{A}_{\mathrm{C}}(\alpha)=\left[\begin{array}{cccc}
0 & 0 & 1 & 0 \\
0 & 0 & 0 & 1 \\
{[a]_{31}} & {[a]_{32}} & {[a]_{33}} & {[a]_{34}} \\
{[a]_{41}} & {[a]_{42}} & {[a]_{43}} & {[a]_{44}}
\end{array}\right]
$$

with

$$
\begin{array}{ll}
{[a]_{31}=[-9.624 ;-0.708] \cdot 10^{3}} & {[a]_{32}=[0.003 ; 0.048] \cdot 10^{3}} \\
{[a]_{33}=[-1.531 ;-0.120] \cdot 10^{3}} & {[a]_{34}=[0.031 ; 0.400] \cdot 10^{3}} \\
{[a]_{41}=[-1.931 ;-1.930] \cdot 10^{3}} & {[a]_{42}=[0.009 ; 0.010] \cdot 10^{3}} \\
{[a]_{43}=[-0.307 ;-0.306] \cdot 10^{3}} & {[a]_{44}=[0.079 ; 0.080] \cdot 10^{3}}
\end{array}
$$

considering the initial uncertainty of $[\alpha](0)=\left[-\frac{\pi}{2}+\epsilon ; \frac{\pi}{2}-\epsilon\right]$.

\section{References}

[1] Ackermann, J. Robust Control - The Parameter Space Approach. SpringerVerlag, London, 2002. DOI: 10.1007/978-1-4471-0207-6.

[2] Aschemann, H., Schindele, D., and Ritzke, J. State and Disturbance Estimation for Robust Control of Fast Flexible Rack Feeders. In Rauh, A. and Auer, E., editors, Modeling, Design, and Simulation of Systems with Uncertainties, Mathematical Engineering, pages 333-351. Springer, Berlin, Heidelberg, 2011. DOI: $10.1007 / 978-3-642-15956-5 \_16$. 
[3] Baumann, W.T. and Rugh, W.J. Feedback Control of Nonlinear Systems by Extended Linearization. IEEE Transactions on Automatic Control, 31(1):4046, 1986. DOI: 10.1109/TAC.1986.1104100.

[4] Duan, G.-R. and Yu, H.-H. LMIs in Control Systems. CRC Press, 2013. DOI: $10.1201 / \mathrm{b} 15060$.

[5] Efimov, D., Raïssi, T., Chebotarev, S., and Zolghadri, A. Interval State Observer for Nonlinear Time Varying Systems. Automatica, 49(1):200-205, 2013. DOI: $10.1016 / \mathrm{j}$.automatica.2012.07.004.

[6] Ifqir, S., Rauh, A., Kersten, J., Ichalal, D., Ait-Oufroukh, N., and Mammar, S. Interval Observer-Based Controller Design for Systems with State Constraints: Application to Solid Oxide Fuel Cells Stacks. In Proc. of 24th International Conference on Methods and Models in Automation and Robotics 2019, Miedzyzdroje, Poland, 2019. DOI: 10.1109/mmar.2019.00000000.

[7] Kaczorek, T. Positive 1D and 2D Systems. Springer-Verlag, London, 2002. DOI: $10.1007 / 978-1-4471-0221-2$.

[8] Kersten, J., Rauh, A., and Aschemann, H. Interval Methods for the Implementation and Verification of Robust Gain Scheduling Controllers. In Proc. of 22nd International Conference on Methods and Models in Automation and Robotics 2017, Miedzyzdroje, Poland, 2017. DOI: 10.1109/mmar.2017.8046929.

[9] Kersten, J., Rauh, A., and Aschemann, H. Interval methods for robust gain scheduling controllers - An LMI-based approach. Granular Computing, 2018. DOI: $10.1007 / \mathrm{s} 41066-018-00147-1$.

[10] Kersten, J., Rauh, A., and Aschemann, H. State-Space Transformations of Uncertain Systems With Purely Real and Conjugate-Complex Eigenvalues Into a Cooperative Form. In Proc. of 23rd International Conference on Methods and Models in Automation and Robotics 2018, Miedzyzdroje, Poland, 2018. DOI: $10.1109 / \mathrm{mmar} .2018 .8486085$.

[11] Kersten, J., Rauh, A., and Aschemann, H. Application-Based Discussion of Verified Simulations of Interval Enclosure Techniques. In Proc. of 24th International Conference on Methods and Models in Automation and Robotics 2019, Miedzyzdroje, Poland, 2019. DOI: 10.1109/MMAR.2019.8864673.

[12] Löfberg, J. YALMIP: A Toolbox for Modeling and Optimization in MATLAB. In Proceedings of IEEE International Symposium on Computer Aided Control Systems Design, pages 284-289, Taipei, Taiwan, 2004. DOI: 10.1109/CACSD . 2004.1393890.

[13] Mazenc, F. and Bernard, O. Asymptotically Stable Interval Observers for Planar Systems With Complex Poles. IEEE Transactions on Automatic Control, 55(2):523-527, 2010. DOI: 10.1109/TAC.2009.2037472. 
[14] Nedialkov, N. S. Interval Tools for ODEs and DAEs. In CD-Proceedings of the 12th GAMM-IMACS International Symposium on Scientific Computing, Computer Arithmetic, and Validated Numerics SCAN 2006, Duisburg, Germany, 2007. IEEE Computer Society. DOI: 10.1109/SCAN.2006.28.

[15] Raïssi, T. and Efimov, D. Some Recent Results on the Design and Implementation of Interval Observers for Uncertain Systems. at-Automatisierungstechnik, 66(3):213-224, 2018. DOI: 10.1515/auto-2017-0081.

[16] Raïssi, T., Efimov, D., and Zolghadri, A. Interval State Estimation for a Class of Nonlinear Systems. IEEE Transactions on Automatic Control, 57:260-265, 2012. DOI: $10.1109 / \mathrm{TAC} .2011 .2164820$.

[17] Rauh, A., Auer, E., and Hofer, E. P. VAlEncIA-IVP: A Comparison with Other Initial Value Problem Solvers. In CD-Proceedings of the 12th GAMMIMACS International Symposium on Scientific Computing, Computer Arithmetic, and Validated Numerics SCAN 2006, Duisburg, Germany, 2007. IEEE Computer Society. DOI: 10.1109/SCAN.2006.47.

[18] Rauh, A., Kersten, J., and Aschemann, H. Techniques for Verified Reachability Analysis of Quasi-Linear Continuous-Time Systems. In Proc. of 24th International Conference on Methods and Models in Automation and Robotics 2019, Miedzyzdroje, Poland, 2019. DOI: 10.1109/MMAR.2019.8864648.

[19] Rauh, A., Westphal, R., and Aschemann, H. Verified Simulations of Control Systems With Interval Parameters Using an Exponential State Enclosure Technique. In Proc. of 18th International Conference on Methods and Models in Automation and Robotics 2013, Miedzyzdroje, Poland, 2013. DOI: $10.1109 / \mathrm{mmar} .2013 .6669913$.

[20] Rauh, A., Westphal, R., Aschemann, H., and Auer, E. Exponential Enclosure Techniques for Initial Value Problems with Multiple Conjugate Complex Eigenvalues. In Nehmeier, M., von Gudenberg, J. Wolff, and Tucker, W., editors, Scientific Computing, Computer Arithmetic, and Validated Numer$i c s$, pages 247-256, Cham, 2016. Springer International Publishing. DOI: 10. 1007/978-3-319-31769-4_20.

[21] Rump, S.M. IntLab - INTerval LABoratory. In Csendes, T., editor, Developments in Reliable Computing, pages 77-104. Kluver Academic Publishers, 1999. DOI: $10.1007 / 978-94-017-1247-7 \_7$.

[22] Scherer, C. and Weiland, S. Linear Matrix Inequalities in Control. In Levine, W. S., editor, Control System Advanced Methods, The Electrical Engineering Handbook Series, pages 24-1-24-30. CRC Press, Boca Raton, 2nd edition, 2011. DOI: $10.1201 / \mathrm{b} 10384$.

[23] Shabana, A.A. Dynamics of Multibody Systems. Cambridge University Press, Cambridge, 2005. DOI: $10.1002 / \mathrm{nme} .1620290812$. 
[24] Smith, H. L. Monotone Dynamical Systems: An Introduction to the Theory of Competitive and Cooperative Systems, volume 41. Mathematical Surveys and Monographs, American Mathematical Soc., 1995. DOI: 10.1090/surv/041.

[25] Sturm, J. F. Using SeDuMi 1.02, A MATLAB Toolbox for Optimization over Symmetric Cones. Optimization Methods and Software, 11-12(1-4):625-653, 1999. DOI: $10.1080 / 10556789908805766$. 